\title{
Response of Ventricular Repolarization Parameters to Preload Changes in the Isolated Working Heart
}

\author{
Jakub Hejc ${ }^{1}$, Oto Janousek ${ }^{1}$, Marina Ronzhina ${ }^{1}$, Tibor Stracina ${ }^{2}$, Veronika Olejnickova ${ }^{2}$, Jana \\ Kolarova $^{1}$, Marie Novakova ${ }^{2,3}$ \\ ${ }^{1}$ Brno University of Technology, Brno, Czech Republic \\ ${ }^{2}$ Masaryk University, Brno, Czech Republic \\ ${ }^{3}$ International Clinical Research Center, Brno, Czech Republic
}

\begin{abstract}
Heart rate dependency of ventricular repolarization (VR) parameters has been a well known phenomenon for many years. Other contributing factors, such as Mechano-electric Coupling, are still under investigation. In this work, we analyze step response of VR parameters after abrupt change in mechanical end-diastolic load.

10 isolated rabbit hearts were perfused in Working Heart mode. The protocol consisted of abrupt changes in preload level of $3 \mathrm{cmH}_{2} \mathrm{O}$. Left ventricle electric activity was recorded by seven unipolar leads at sampling frequency $10 \mathrm{kHz}$. A second-order transfer function model was used to identify basic parameters of $Q T_{E N D}$ and $Q T_{P E A K}$ step responses, detected in reconstructed global pseudo-lead.

Results show inverse relationship between changes in the preload and the length of $Q T$ related intervals. Increase of the preload causes significant $(p<0.05)$ shortening of both intervals within the range of 1.6$26.9 \mathrm{~ms}$. On the contrary, preload decreasing leads to prolongation of repolarization parameters, however, with significantly lower $(p<0.05)$ average magnitude. Dynamic response is highly individual among each heart. Average time needed to reach stable QT in our data is 336.0 seconds, while min. and max. value is 41.4 and 894.0 seconds, respectively.
\end{abstract}

\section{Introduction}

Heart rate dependency of ventricular repolarization (VR), mainly represented by QT interval, is a well-known phenomenon, and has been largely studied since many year ago [1], [2], [3]. Other contributing factors, which could clarify the regulatory mechanism of VR, are still under investigation. One of possible mechanisms in an intrinsic link between mechanical and electrical activity of the heart, so-called Mechano-electric Coupling [4]. Stretch-induced changes in the left ventricle (LV) electric activity has been previously studied in [5] using a waterfilled balloon inserted into the LV of Langendorffperfused heart. In this work, we investigate the dynamic response of VR parameters to abruptly increased and decreased preload in an isolated working heart model.

\section{Material and methods}

All animal experiments were carried out with respect to recommendations of the European Community Guide for the Care and Use of Laboratory Animals. The experimental protocol was approved by local Committee for Ensuring the Welfare of Laboratory Animals.

\subsection{Experimental setting}

Ten adult New Zealand white rabbits (both sexes) were medicated by diazepam (2.5 mg), xylazin $(4 \mathrm{mg} / \mathrm{kg})$, ketamin $(60 \mathrm{mg} / \mathrm{kg})$ and heparin $(1000 \mathrm{IU} / \mathrm{kg})$. The heart was excised and fixed to a modified Working Heart System (Radnoti, USA). The heart was perfused with Krebs-Henseleit solution $\left(2.5 \mathrm{mM} \mathrm{CaCl} 2,37^{\circ} \mathrm{C}, \mathrm{pH}\right.$ 7.4) aerated by pneumoxyd $\left(95 \% \mathrm{O}_{2} ; 5 \% \mathrm{CO}_{2}\right)$.

For 15 min., heart was perfused in Langendorff mode at constant pressure $(80 \mathrm{mmHg})$. System was then switched to Working Heart mode (preload, $8 \mathrm{cmH}_{2} \mathrm{O}$; afterload, $60 \mathrm{cmH}_{2} \mathrm{O}$ ). The heart was allowed to stabilize for next $15 \mathrm{~min}$.

During the protocol (Figure 1), the heart rate was controlled through pacing the right atrium to inhibit the influence of QT/RR relationship. Baseline of the pacing rate was set at $10 \%$ above natural heart rate. Single upward and downward step in the heart rate was included to verify correct dynamic response of QT/RR. Preload 
and afterload were adjusted by appropriate height of the water column. The step size in all parameters was chosen in order to induce sufficient change in VR parameters while maintaining physiological function of the heart (i.e. satisfactory cardiac output, avoiding EG abnormalities).

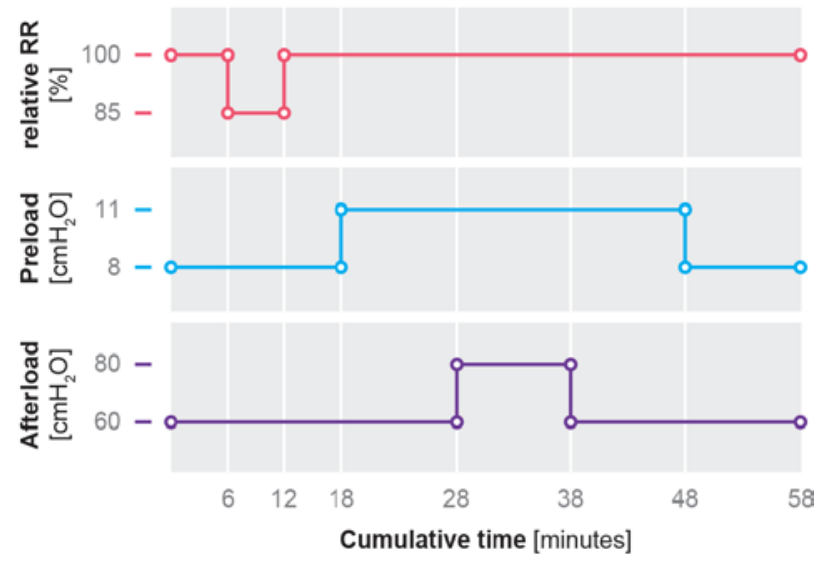

Figure 1. Schedule of the experimental protocol.

\subsection{Data recording}

Unipolar electrograms (EGs) were measured by seven non-contact [6] Ag-AgCl electrodes placed on an interior wall of buffer-filled chamber. Electrodes were uniformly spaced along a semicircle so that electrical activity of the entire LV was recorder.

Measurement of LA volumetric flow rate was performed by inline ultrasonic flow meter (Transonic Systems, USA). Signals were amplified by DAM50 BioAmplifiers (World Precision Instruments, USA), and acquired by NI USB-6259 measuring card (National Instruments, USA) with sampling frequency $10,000 \mathrm{~Hz}$ and 16-bit resolution.

\subsection{Signal processing}

To reduce high frequency noise, all EGs were preprocessed with a low-pass Butterworth filter $\left(10^{\text {th }}\right.$ order, $500 \mathrm{~Hz}$ cut-off frequency) in both, forward and reverse direction. Cubic spline interpolation was used for the baseline wander correction.

All of the 7 unipolar pre-processed EGs were then subjected to Singular Value Decomposition (SVD) obtaining new set of 7 transformed pseudo-leads $\boldsymbol{w}_{i}(n)$. Multidimensional signal was reduced to a onedimensional global pseudo-lead $\boldsymbol{w}_{G}(n)$ :

$$
w_{G}(n)=\sqrt{w_{1}(n)^{2}+w_{2}(n)^{2}},
$$

where $\boldsymbol{w}_{1}(n)$ and $\boldsymbol{w}_{2}(n)$ are the first two eigenvectors.
The signal $\boldsymbol{w}_{\mathrm{G}}(n)$ was analyzed by a modified custommade algorithm [7] in order to obtain position of the QRS complex onset, $\mathrm{R}$ wave, $\mathrm{T}$ wave peak and $\mathrm{T}$ wave offset in all valid beats. Detected positions were verified and corrected manually. Continuous series of $\mathbf{Q T}_{\mathrm{END}}$ and $\mathbf{Q T}_{\text {PEAK }}$ intervals were calculated as representatives of global repolarization.

Beats with artifacts or unclear position of one of the fiducial points were excluded from further investigation. In some parts of the signal, QT variability could not be determined due to low signal to noise ratio. These segments were also excluded from the analysis.

\subsection{Analysis of QT dynamic response}

Based on the oscillatory nature of the response data, a second-order transfer function ( $\mathrm{TrF}$ ) model with one zero and time delay was used to identify dynamic parameters of the preload related QT changes. The time delay was needed to compensate slow response after step initiation and to reduce the model order.

Following parameters were estimated in all valid step responses:

1. Dead time $\tau_{\mathrm{D}}$ : Time delay of the model.

2. Rise time $\boldsymbol{\tau}_{\mathrm{R}}$ : The time required for the response to rise from 10 to $90 \%$ of its steady state value $y(\infty)$.

3. Settling time $\boldsymbol{\tau}_{\mathrm{s}}$ : The period required for the output to fall within the interval $y(\infty) \pm 2 \%$.

4. Peak amplitude $\mathbf{A}_{\mathrm{P}}$ : The amplitude of the $1_{\text {st }}$ peak.

5. DC gain $\mathbf{G}_{\mathrm{DC}}$ : Difference between $y(\infty)$ and $y(0)$.

6. Damping ratio $\zeta$ : The measure of the model oscillation tendency.

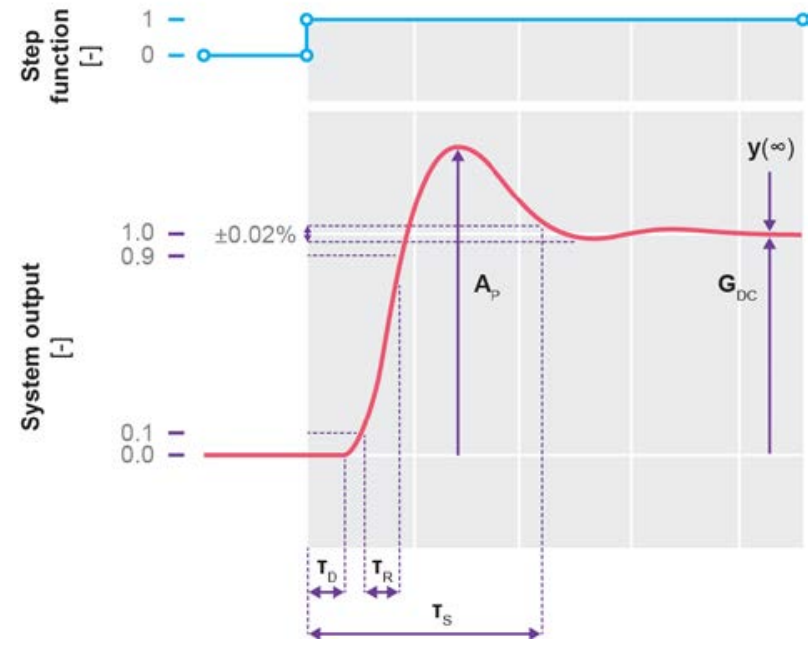

Figure 2. Basic parameters for evaluation of the dynamic response of the second-order system (red line). Blue line represents the Heaviside step function. 


\section{Results}

Table 1 shows the summary results of $\mathrm{QT}_{\mathrm{END}}$ dynamic parameters, estimated separately for increase and decrease in LV load. Table 2 shows the corresponding results of the QT $\mathrm{T}_{\mathrm{PEAK}}$ parameters.

Examples of typical responses of QT related intervals are presented in Figures 3-4. Smooth line over the experimental data corresponds to the output of the fitted model. Parameter InFL-Vol (bottom blue waveform) is the total volume flowing into the heart during complete heart cycle. There are two different shapes of an adaptation process, which can be classified by damping ratio $\zeta$. The response of critically damped system with $\zeta=1$ are depicted in the upper half, while the underdamped response with $\zeta<1$ is shown in the lower half of Figure 3 and 4. Considering $\mathrm{QT}_{\mathrm{END}}$ and $\mathrm{QT}_{\mathrm{PEAK}}$ adaptation, there were only 6 , respectively 4 identified models with $\zeta=1$. In one heart, repeated protocol reveals both types of responses within the same experimental phase.
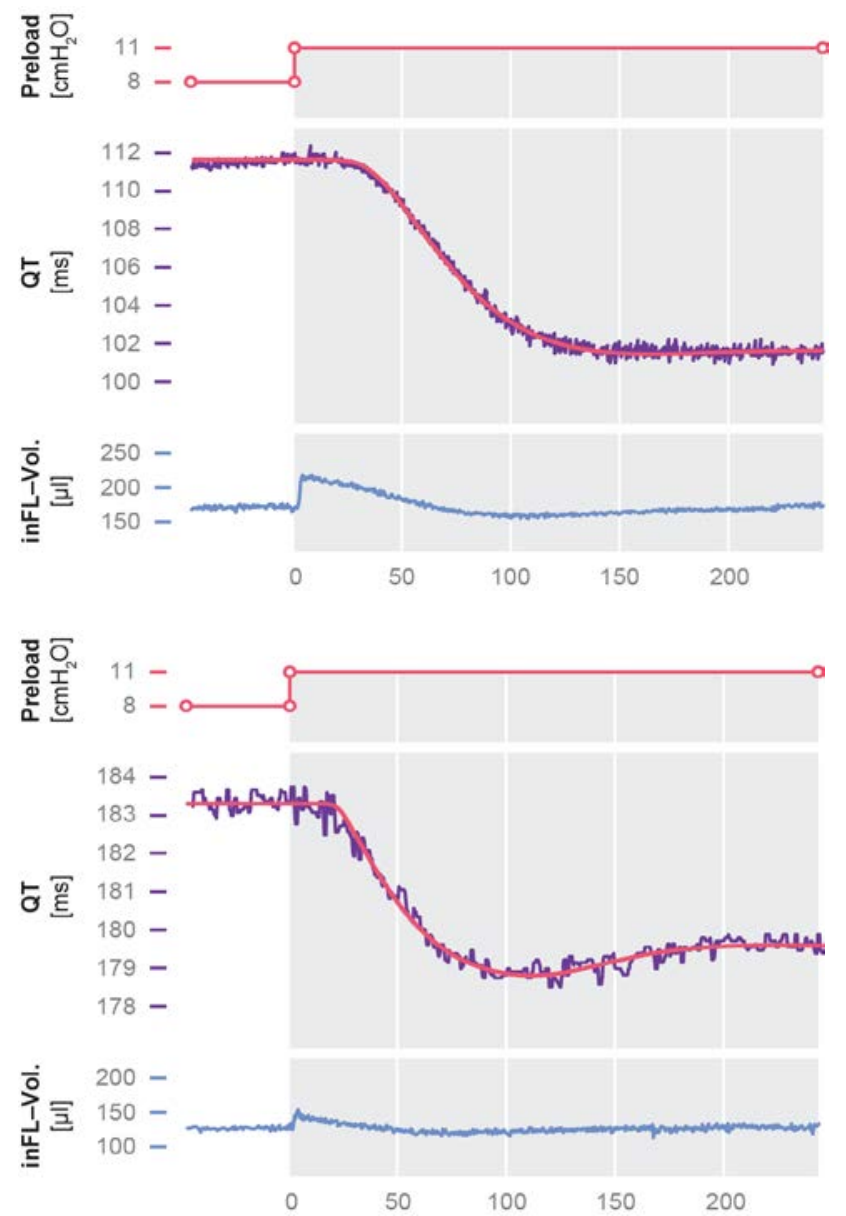

Figure 3. Increase in LV hemodynamic load (red line) followed by shortening of $\mathrm{QT}_{\mathrm{END}}$ and $\mathrm{QT}_{\mathrm{PEAK}}$ interval (purple line).
Table 1. Summary of the $\mathrm{QT}_{\mathrm{END}}$ dynamic parameters.

\begin{tabular}{lll}
\hline Parameter & $\begin{array}{l}\uparrow \text { Preload } \\
(\text { mean } \pm \text { SD) }\end{array}$ & $\begin{array}{l}\downarrow \text { Preload } \\
(\text { mean } \pm \text { SD) }\end{array}$ \\
\hline$\tau_{\mathrm{D}}[\mathrm{s}]$ & $7.2 \pm 5.8$ & $5.7 \pm 9.0$ \\
$\tau_{\mathrm{R}}[\mathrm{s}]$ & $39.2 \pm 19.9$ & $63.0 \pm 25.4$ \\
$\tau_{\mathrm{S}}[\mathrm{s}]$ & $360.8 \pm 196.9$ & $314.8 \pm 208.2$ \\
$\mathrm{G}_{\mathrm{DC}}[\mathrm{ms}]$ & $-8.3 \pm 6.8$ & $5.4 \pm 3.0$ \\
$\mathrm{~A}_{\mathrm{P}}[\mathrm{ms}]$ & $13.5 \pm 9.8$ & $7.4 \pm 4.3$ \\
\hline
\end{tabular}

Table 2. Summary of the $\mathrm{QT}_{\mathrm{PEAK}}$ dynamic parameters.

\begin{tabular}{lll}
\hline Parameter & $\begin{array}{l}\uparrow \text { Preload } \\
(\text { mean } \pm \text { SD) }\end{array}$ & $\begin{array}{l}\downarrow \text { Preload } \\
(\text { mean } \pm \text { SD) }\end{array}$ \\
\hline$\tau_{\mathrm{D}}[\mathrm{s}]$ & $11.5 \pm 7.6$ & $8.4 \pm 8.3$ \\
$\tau_{\mathrm{R}}[\mathrm{s}]$ & $33.7 \pm 20.6$ & $85.3 \pm 63.8$ \\
$\tau_{\mathrm{S}}[\mathrm{s}]$ & $382.9 \pm 259.6$ & $385.3 \pm 169.2$ \\
$\mathrm{G}_{\mathrm{DC}}[\mathrm{ms}]$ & $-11.1 \pm 7.9$ & $8.8 \pm 5.4$ \\
$\mathrm{~A}_{\mathrm{P}}[\mathrm{ms}]$ & $17.8 \pm 13.2$ & $11.8 \pm 6.9$ \\
\hline
\end{tabular}
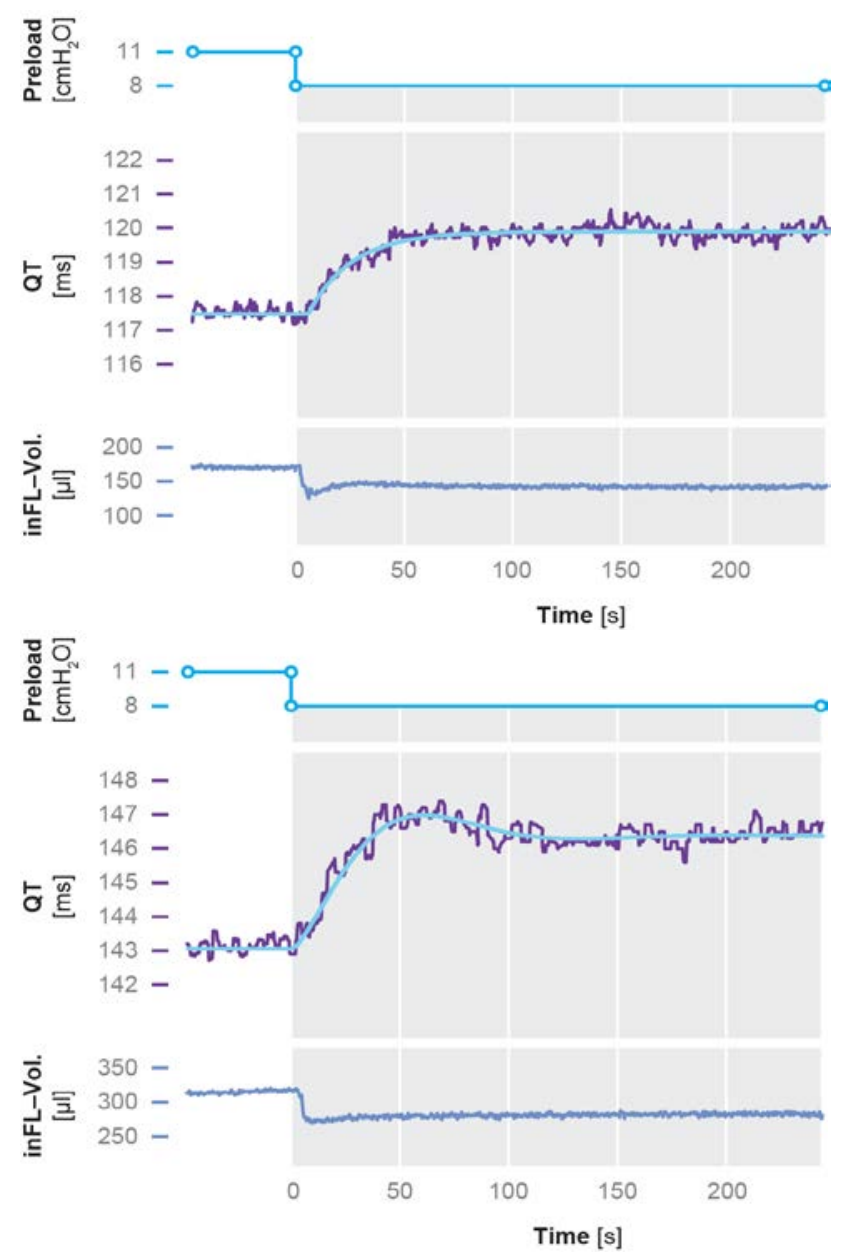

Figure 4. Decrease in LV hemodynamic load (red line) followed by shortening of $\mathrm{QT}_{\mathrm{END}}$ and $\mathrm{QT}_{\mathrm{PEAK}}$ interval (purple line). 


\section{Discussion}

The results show clear inverse relationship between changes in preload and the length of $\mathrm{QT}_{\mathrm{END}}$ and $\mathrm{QT}_{\mathrm{PEAK}}$ interval. Increase in the LV load causes significant $(\mathrm{p}<0.05)$ shortening of both, $\mathrm{QT}_{\mathrm{END}}$ and $\mathrm{QT}$ PEAK. Here, average difference between default level and new stable state of $\mathrm{QT}_{\mathrm{END}}$ and $\mathrm{QT}_{\mathrm{PEAK}}$, given by parameter $\mathrm{G}_{\mathrm{DC}}$, is $-8.8 \pm 6.9$ and $-11.1 \pm 7.9$, respectively. Furthermore, $\mathrm{G}_{\mathrm{DC}}$ in $\mathrm{QT}_{\text {PEAK }}$ shortens more $(\mathrm{p}<0.05)$ than $\mathrm{QT}_{\text {END. }}$.

Conversely, decrease in preload leads to prolongation of $\mathrm{QT}_{\mathrm{END}}\left(\mathrm{G}_{\mathrm{DC}}=5.4 \pm 3.0\right)$ and $\mathrm{QT}_{\mathrm{PEAK}}\left(\mathrm{G}_{\mathrm{DC}}=8.8 \pm 5.4\right)$. In this case, $G_{D C}$ is significantly higher $(p<0.05)$ within

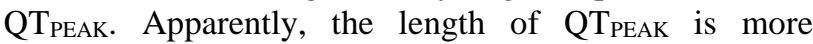
variable in reaction to step change in LV load in both of the directions.

Parameters $\tau_{\mathrm{D}}, \tau_{\mathrm{R}}$ and $\tau_{\mathrm{S}}$ show large inter-individual profile of the adaptation process. In most of the cases, the process is delayed $\left(\tau_{\mathrm{D}}\right)$ by $5-27$ seconds. Parameter $\tau_{R}$ is significantly shorter $(\mathrm{p}<0.05)$ in $\mathrm{QT}_{\mathrm{END}}$ after increase $(39.2 \pm 19.9$ s.) than after decrease $(63.0 \pm 25.4$ s.) in $\mathrm{LV}$ load. Similar trend can be observed also in $\mathrm{QT}_{\text {PEAK }}$ without statistical significance. Average time needed to reach stable QT considering the entire dataset is 336.0 seconds. However, observed values vary between 41.4 and 894.0 seconds.

The second-order $\operatorname{TrF}$ model used in this work is suitable for estimating elementary dynamic parameters due to oscillatory nature of QT response. However, loworder models suppress low-amplitude oscillations that may precede a major uptake.

\section{Conclusion}

Ventricular repolarization is affected by change in left ventricle load. The length of $\mathrm{QT}_{\mathrm{PEAK}}$ and $\mathrm{QT}_{\mathrm{END}}$ interval is inversely proportional to the preload level in working isolated heart.

Considering the time-dependent changes InFL-Vol index, it is obvious that LV hemodynamic parameters are also adapted after performing the step in LV load. Hemodynamic and mechanical properties of the ventricle should be taken into consideration to fully understand intrinsic mechanism of mechano-electric coupling at a whole-organ level.

\section{Study limitations}

Influence of the heart rate baseline on the QT dynamic parameters has not been taken into consideration in this study and will be subjected to further investigation in future work.

\section{Acknowledgements}

This work was supported by grant project of the Grant Agency of the Czech Republic GAP 102/12/2034 and Specific University Research Grant of Masaryk University no. MUNI/A/1326/2014, as provided by the Ministry of Education, Youth and Sports of the Czech Republic in the year 2015.

\section{References}

[1] Lau C, Freedman AR, Fleming S, Malik M, Camm AJ, Ward DE. Hysteresis of the Ventricular Paced QT Interval in Response to Abrupt Changes in Pacing Rate. Cardiovascular Research. 1988;22:67-72.

[2] Malik M, Hnatkova K, Sisakova M, Schmidt G. Subjectspecific Heart Rate Dependency of Electrocardiographic QT, PQ, and QRS intervals. Journal of Electrocardiology, 2008;41: 491-497.

[3] Halamek J, Jurak P, Lipoldova J, Leinveber P. QT/RR Coupling and Gender Differences. Computing in Cardiology 2010;37:365-368.

[4] Quinn TA, The Importance of Non-uniformities in Mechano-Electric Coupling for Ventricular Arrhythmias. J Interv Card Electrophysiol 2014;39:25-35.

[5] Zabel M, Koller BS, Sachs F, Franz MR. Stretch-induced Voltage Changes in the Isolated Beating Heart: Importance of the Timing of Stretch and Implications for StretchActivated Ion Channels. Cardiovascular Research 1996;32:120-130.

[6] Kolářová J, Fialová K, Janoušek O, Nováková M, Provazník I. Experimental Methods for Simultaneous Measurement of Action Potentials and Electrograms in Isolated Heart. Physiol Res 2010;59(S1):71-80

[7] Hejč J, Vítek M, Ronzhina M, Nováková M, Kolářová J. A Wavelet-Based ECG Delineation Method: Adaptation to an Experimental Electrograms with Manifested Global Ischemia. Cardiovasc Eng Technol 2015;6:364-75

Address for correspondence:

Jakub Hejc

Department of Biomedical Engineering, Faculty of Electrical Engineering and Communication, Brno University of Technology,

Technická 12, 61600 Brno, Czech Republic. xhejcj00@stud.feec.vutbr.cz 